\title{
A Novel Microflow Phantom Dedicated to Ultrasound Microvascular Measurements
}

\author{
Virginie Grand-Perret ${ }^{1}$, Jean-René Jacquet ${ }^{2}$, Ingrid Leguerney ${ }^{1,3}$, Baya Benatsou ${ }^{1,3}$, Jean-Marc \\ Grégoire $^{2}$, Georges Willoquet ${ }^{1}$, Ayache Bouakaz ${ }^{2}$, Nathalie Lassau ${ }^{1,3}$, and Stephanie Pitre- \\ Champagnat $^{1}$ \\ ${ }^{1}$ Imagerie par Resonance Magnetique Medicale et Multi-Modalites, Université Paris-Saclay, Orsay, France \\ 2Imagerie et cerveau, Inserm, Univ. François Rabelais, Tours, France \\ ${ }^{3}$ Research Department, Gustave Roussy Cancer Campus, Université Paris-Saclay, Villejuif, France \\ Corresponding Author: \\ Virginie Grand-Perret, Imagerie par Resonance Magnetique Medicale et Multi-Modalites, UMR8081, Bâtiment 220, \\ Université Paris-Sud, 91405 Orsay, France. \\ Email: virginie.grand-perret@u-psud.fr
}

\begin{abstract}
Tumor microvascularization is a biomarker of response to antiangiogenic treatments and is accurately assessed by ultrasound imaging. Imaging modes used to visualize slow flows include Power Doppler imaging, dynamic contrast-enhanced ultrasonography, and more recently, microvascular Doppler. Flow phantoms are used to evaluate the performance of Doppler imaging techniques, but they do not have a steady flow and sufficiently small channels. We report a novel device for robust and stable microflow measurements and the study of the microvascularization. Based on microfluidics technology, the prototype features wall-less cylindrical channels of diameters ranging from as small as 147 up to $436 \mu \mathrm{m}$, cast in a soft silicone polymer and perfused via a microfluidic flow pressure controller. The device was assessed using flow rates from 49 to $146 \mu \mathrm{L} / \mathrm{min}$, with less than $1 \%$ coefficient of variation over three minutes, corresponding to velocities of 6 to $142 \mathrm{~mm} / \mathrm{s}$. This enabled us to evaluate and confirm the reliability of the Superb Microvascular Imaging Doppler mode compared with the Power Doppler mode at these flow rates in the presence of vibrations mimicking physiological motion.
\end{abstract}

\section{Keywords}

ultrasound microvasculature, microfluidics, flow phantom, flow imaging, microvascular Doppler, superb microvascular imaging, power Doppler imaging

\section{Introduction}

Assessment of microvascularization in tissues is clinically relevant in cardiology ${ }^{1,2}$ and oncology. ${ }^{3}$ Angiogenesis is among the key processes involved in tumor growth. ${ }^{4}$ As a therapeutic target for antiangiogenic drugs, angiogenesis is also a promising biomarker for the evaluation of patient response to treatment. ${ }^{5}$ 
In this context, ultrasound imaging is an attractive technique for exploring flow in vascular networks at different scales. Color Doppler and pulsed Doppler modes are usually used to image fast flows, while microvascular flows are typically imaged using Power Doppler mode (PD), which is able to visualize perfusion in vessels with diameters of a few hundred micrometers. ${ }^{6}$ Microvasculature assessment in vessels below the limit of a few hundred micrometers can be performed by specific modes. Dynamic contrast-enhanced ultrasound (DCE-US), which is based on the injection of contrast agents (encapsulated microbubbles) to enhance the scattered signal, enables quantification of the microvasculature. New microvascular Doppler modes based on advanced signal processing both in "conventional" imaging as well as in ultrafast plane-wave imaging were recently developed to visualize microvascularization without the need for contrast agent injection..$^{7-10}$ In particular, Superb Microvascular Imaging ${ }^{\circledR}$ (SMI) available on Aplio 500 (Toshiba Medical Systems, Japan) is based on high-resolution Doppler imaging with high-density beamformer architecture and a powerful and adaptive algorithm that effectively separates flow signals from tissue motion artifacts.

Phantoms are commonly used to characterize and calibrate ultrasound imaging systems and to verify whether their performance meets quality standards. ${ }^{11}$ They are also used to assist the development of new transducers and diagnostic techniques, as well as to assess new imaging modalities and image processing techniques. Moreover, they can be used to demonstrate clinical improvements. ${ }^{12}$ Specific flow phantoms have been developed to perform reproducible tests to evaluate the performance of new microvascular flow imaging modalities and microflow Doppler image processing techniques. The phantoms described below are test objects made out of tissuemimicking materials (TMM) with acoustic properties mimicking those of human tissues and vessels, specified by the International Electrotechnical Commission $61685 .{ }^{11}$ The geometries are mainly straight channels, given the fact that they study the resolution and detection limits of the scanners and their associated imaging modes. More tortuous geometries that are more physiologically relevant would be interesting to assess quantitative imaging realistically, with wash-in and wash-out phases that would be sensitive to the tortuous geometry of the flow : such phantoms have been made for three-dimensional (3D) ultrasound flow imaging, using rapid prototyping and investment casting techniques ${ }^{13}$ as well as lost-core casting principles ${ }^{14}$ to assess the efficacy of ultrasound flow estimation methods.

Doppler flow phantoms with a channel of 1 to $2 \mathrm{~mm}$ can be made by casting TMM around a rubber tube, ${ }^{15}$ or around a rod that is removed after solidification of the TMM to obtain a wallless design. ${ }^{16}$ However, blood capillary diameters are typically 5 to $500 \mu \mathrm{m},{ }^{17}$ so microvascularization phantoms need to contain thinner channels. Thinner tubes of 200 to $700 \mu \mathrm{m}$-diameter can also be encapsulated in TMM,${ }^{18}$ however TMM has a limited shelf life (a few months) and the wall around the channel can cause reflection artifacts. To downsize the channel, a dialysis cartridge composed of a large number of cellulose capillaries of $200 \mu \mathrm{m}$-diameter has been used, ${ }^{19}$ however the capillaries become porous over time, and only the top capillaries can be imaged because of ultrasound attenuation. This setup is thus not reproducible, and capillaries cannot be imaged individually.

New approaches including the use of microfluidic chips ${ }^{20,21}$ appear to be a promising solution to mimic complex microvascular networks. These chips allow manipulation of fluids in channels ranging from less than a micrometer to a few millimeters, including complex geometries. They possess valuable properties such as slow and laminar flows, wall-less channels and the possibility of coating them with endothelial cells for biological tests. They are easy to prepare in a clean room environment, inexpensive and easily molded. Desailly et al. and Kim et al. ${ }^{20,21}$ used standard polydimethylsiloxane (PDMS) microfluidic chips with rectangular channels to test their ultrasound imaging modes. However, PDMS material is highly absorbent which limits the propa- 
gation of ultrasound waves to superficial depths only.
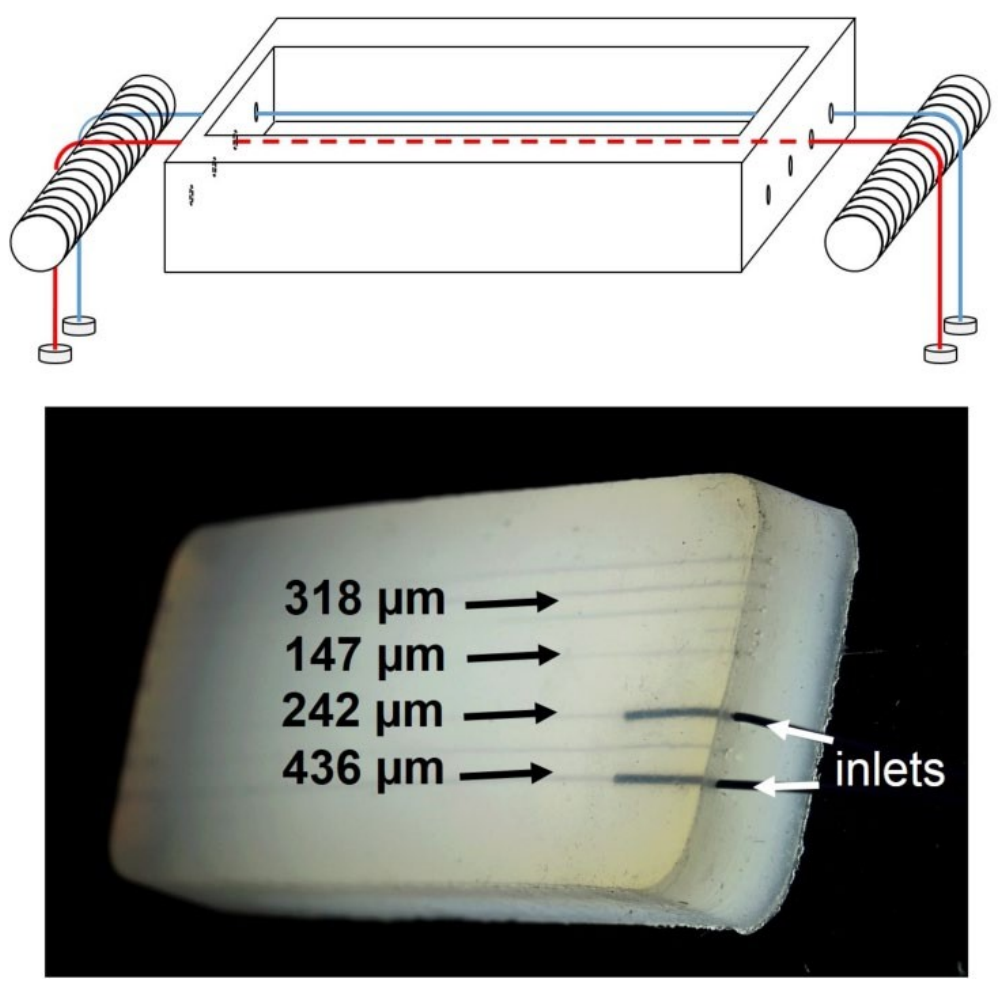

Figure 1. Top: scheme of the process used to make the mold. Strings (red and blue) were placed through the holes of the tank and ballasted at both ends to straighten them. This setup allowed straight strings parallel to the bottom of the mold. Bottom: homogeneous phantom composed of channels including the four channels of diameters $147,242,318$, and $436 \mu \mathrm{m}$. PEEK tubes are plugged into the channel inlets, shown by the white arrows.

The aim of this study was to develop a new generation of microflow phantoms. To this purpose, the first objective was to identify a material less absorbing that PDMS and with a simple fabrication process so that we could make a microfluidic chip without photolithography in a clean room environment. In addition, this new phantom was connected to a setup that precisely controlled the microflow within the channels over time. Flow variability during acquisitions has also been studied. Finally, the potential interest of this new device was illustrated by comparing three microvascular Doppler imaging modes.

\section{Materials and Methods}

\section{Phantom Design}

Ecoflex 00-30, a soft silicone, was cast into a $2.5 \times 5 \mathrm{~cm}$ custom-made mold. Four holes of 500 $\mu \mathrm{m}$-diameter, spaced 2 to $5 \mathrm{~mm}$ were drilled from either side in alignment between each border of the mold. They were placed $2 \mathrm{~mm}$ from the bottom of the mold (the top of the phantom) to place the channels $2 \mathrm{~mm}$ from the incident surface (Figure 1). Strings were placed through the holes, ballasted at both ends, and guided by the screw threads. This setup allowed straight strings parallel to the bottom of the mold. The channels were then prepared using different diameters 
strings: $436 \mu \mathrm{m}$ metal fishing line, 318 nylon fishing line, 147 and $242 \mu \mathrm{m}$ sutures (monocryl 4-0 and monocryl 5-0). The diameter sizes were measured optically with an AZ-100 multizoom macroscope (Nikon, Japan) and the values indicated are the means of the two extremum values observed with the macroscope along the string. The macroscope supplier's software (Nikon, Japan) gave measurements with a $0.01 \mu \mathrm{m}$ precision.

Once the strings were in place, the Ecoflex 00-30 material was cast with a thickness of $1 \mathrm{~cm}$ after degassing in a vacuum chamber for five to 10 minutes to remove air bubbles. It was left for four hours at room temperature for complete curing of the polymer. Finally, the strings were removed from the mold. The resulting phantom was a $2.5 \times 5 \times 1 \mathrm{~cm}^{3}$ block composed of seven cylindrical straight channels of diameters ranging from 147 to $436 \mu \mathrm{m}$ (Figure 1), including the four channels we are going to study here.

For microflow experiments, we used a blood-mimicking fluid (BMF) consisting of small particles which mimic ultrasound scatterers such as red blood cells, that are essential in microvascular Doppler modes. The home-made $\mathrm{BMF}^{22-24}$ was composed of nylon beads (Orgasol, Arkema, France) of $5 \mu \mathrm{m}$-diameter mixed with distilled water (83.86\%), glycerol $(10.06 \%$, Merck, Germany), 185000 D dextran (3.36\%, Sigma-Aldrich, Switzerland), and ICI synperonic $\mathrm{N}$ surfactant $(0.9 \%$, BDH, England). Before each use, BMF was filtered through a paper sieve with $30 \mu \mathrm{m}$ pores (Whatman d113, GE Healthcare, UK) with a Büchner device to remove clots that could block the phantom channels.

\section{Materials for Acoustic Characterization}

Six platinum-cure silicones were chosen as they are robust and give good mechanical support, despite their acoustical attenuation: Ecoflex ${ }^{\circledR} 00-10$, Ecoflex 00-20, Ecoflex 00-30, Dragonskin ${ }^{\circledR}$, Sortaclear ${ }^{\circledR}$ 18, and Moldstar ${ }^{\circledR}$ (Smooth-On, Macungie, Pennysylvania, USA). These materials were made following the supplier's instructions with very similar fabrication processes, namely 1:1 ratio and five minutes degassing at a pressure under 29 inches of mercury. The silicones were placed in a vacuum chamber connected to a N145.1.2AT.18 diaphragm vacuum pump (KNF, Germany; Maximum pressure: 7 bars, Delivery rate: $50 \mathrm{~L} / \mathrm{min}$, Ultimate vacuum: $100 \mathrm{mbar}$ ) and degassed five minutes to remove entrapped air. They were then compared so that the least attenuating material was chosen. They differed in hardness and chemical composition. Their acoustical properties were measured using a broadband substitution technique $\mathrm{e}^{25-27}$ on $2 \mathrm{~mm}$-thick silicone samples as shown in Figure 2.

Each sample was placed in front of a broadband 15-30 MHz composite piezoelectric transducer (Imasonic, Voray-sur-l'Ognon, France). A high acoustic impedance stainless steel piece was placed beneath the sample, acting as a perfect reflector: the reflection is considered total. The transducer was used in pulse echo mode, acquiring the backscattered signal. First, the $s_{2}$ and $s_{3}$ signals were acquired, $s_{2}$ being the signal backscattered from the front of the sample and $s_{3}$ being the signal reflected from the interface sample/stainless steel at the back of the sample. Second, the $s_{1}$ signal was acquired independently, without the sample.

Using the substitution method, the speed of sound $V_{s}$ in the sample was calculated using:

$$
V_{s}\left(T^{\circ}\right)=\frac{V_{w}\left(T^{\circ}\right) \cdot\left(t_{1}-t_{2}\right)}{\left(t_{3}-t_{2}\right)},
$$


with $V_{w}$ being the speed of sound in water at a temperature $T^{28}, t_{1}, t_{2}$, and $t_{3}$ were the times of flight corresponding to maximum magnitude of the received signals $s_{1}(t), s_{2}(t)$, and $s_{3}(t)$, respectively.

The density of the sample $\rho$ was measured using the Archimedes principle and the acoustic impedance $Z_{s}$ of the material was calculated using:

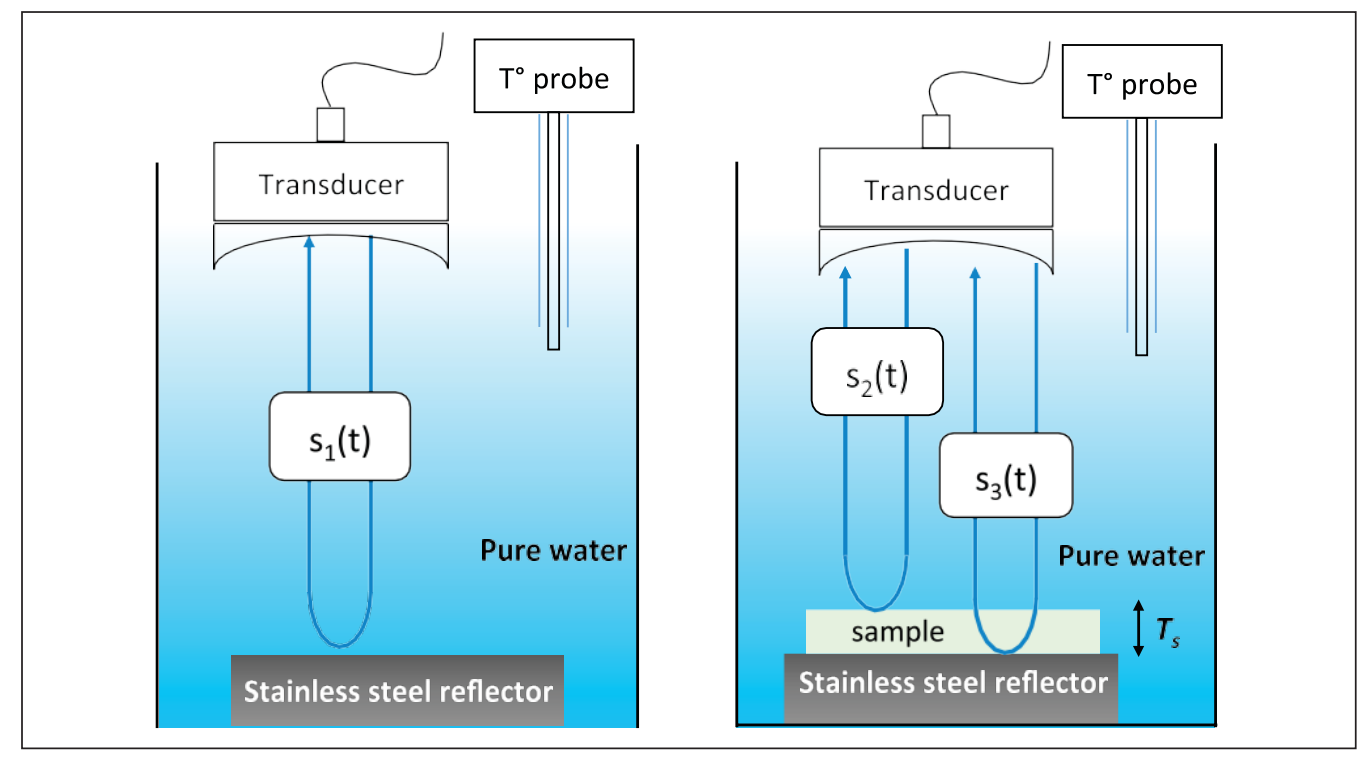

Figure 2. Pulse-echo substitution technique used to characterize the sample acoustical properties. $s_{1}, s_{2}$, and $s_{3}$ are the sound waves echoed on the stainless steel reflector alone $\left(s_{1}\right)$, the sample alone $\left(s_{2}\right)$, and on the stainless steel reflector after going through the sample $\left(s_{3}\right)$. The water temperature is measured with a thermometer to estimate the speed of sound in water. $T_{s}$ is the sample thickness.

$$
Z_{s}=\rho \cdot V_{s} .
$$

The attenuation $\alpha_{s}$ of the sample was calculated with the following equation ${ }^{29}$ :

$$
\alpha_{s}=\frac{20 \log _{\mid}\left(\left|\frac{S_{1}}{S} \times A\right|\right)}{2 T_{s}},
$$

where $S_{1}\left(S_{3}\right)$ is the Fourier transform of the signal $s_{1}\left(s_{3}\right), A$ is the total amplitude transmission coefficient, taking into account the transmit and return transmission values at the interface water/ sample, and $T_{s}$ is the thickness of the sample extracted from the previous calculated speed of sound in the sample using:

$$
T_{s}=V_{s}\left(T^{\circ}\right) \cdot\left(t_{3}-t_{2}\right)
$$


For imaging experiments, the phantom was placed in a tank filled with degassed water and layered with absorbing material (Precision Acoustics, UK). A frame of absorbing material was used to maintain the phantom in the bottom, holding it from floating. The BMF was circulated by a MFCS-EZ microfluidic flow control system (Fluigent SA, France) used as a pressure-based flow controller. This device, commonly used in the microfluidic field, allows the circulation of fluids by pressure regulation. The MFCS operates over a wide pressure range, up to seven bars. To optimize precision, a high resistance circuit was placed upstream to adapt the pressure range displayed by the flow control system. The upstream circuit was composed of $160 \mathrm{~cm}$ of $200 \mu \mathrm{m}$ 


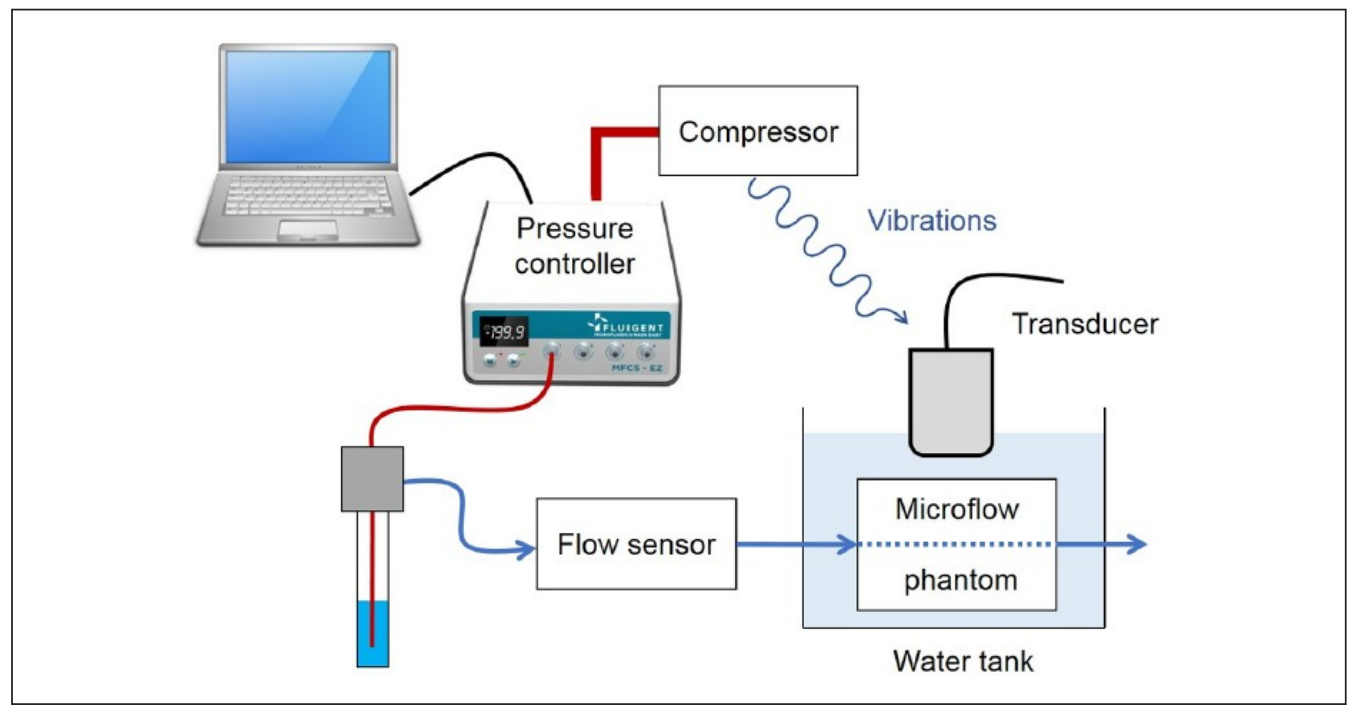

Figure 3. Experimental setup: compressor, pressure controller controlled by Maesflow (Fluigent) software, flow sensor, and microflow phantom.

inner diameter plastic tubes followed by $48 \mathrm{~cm}$ of $250 \mu \mathrm{m}$ inner diameter plastic tubes. The MFCS-EZ system controlled the constant flow of fluid volume in the micro and nano-liter range.

The setup was composed of a compressor, a manual pressure regulator and an air dryer module, the MFCS-EZ, and the reservoir (Figure 3). BMF was put in the reservoir, and connected to the phantom via plastic tubes. PEEK tubes of $250 \mu \mathrm{m}(0.01 \mathrm{inch})$ inner diameter and $0.79 \mathrm{~mm}$ (1/32 inch) outer diameter (IDEX Health \& Science, Rohnert Park, California) are plugged into the channel inlets and outlets, and held in place by the silicone elasticity. A flow-rate platform measures the flow rate in real-time using a thermal sensor. Four different overpressure values were applied to each channel to obtain velocities between 6 and $142 \mathrm{~mm} / \mathrm{s}: 1500,2000,3000$, and 4000 mbar. The flow rate was controlled in real-time with the flow-rate platform. This pressure range was adequate for studying velocities in the range of physiological blood velocity.

A Toshiba Aplio 500 ultrasound scanner (Toshiba Medical Systems, Tokyo, Japan) equipped with a $12 \mathrm{MHz}$ linear array probe $1204 \mathrm{BT}$ was used to perform imaging sequences. The center of the channel was placed at the optimal focal depth of the probe $(1.3 \mathrm{~cm})$. Vibrations were applied on the tank to simulate the physiological motions which are a source of image artifacts. The vibrations originated from the compressor, which was not working in continuous mode: the compressor stopped working when the pressure in the circuit was high enough, and started working again when the pressure was below a certain level. We thus used the compressor as a reproducible vibration source, and we could start the vibrations by opening the pressure circuit. The vibrations were measured using a tri-axis KX023-1025 accelerometer (Kionix, Ithaca, New York, USA; Sensitivity: 16 to 16384 counts/g) fixed on the setup.

\section{Ultrasound Data Acquisition}

Flow was measured over time by the flow-rate platform for a given overpressure in the channels. For the four different pressures and the four channels sizes, 10-second video clips were acquired using Power Doppler Mode (PD), and SMI modes, both with and without the vibrations. Each video clip was rated by two physicists and a small animal U.S. imaging specialist, as figured in Table 2. 
Table 1. Acoustical Properties of Silicones, Soft Tissues, TMM and BMF Using a Broadband Substitution Technique.

\begin{tabular}{|c|c|c|c|c|c|}
\hline & Density (g/cc) & $\begin{array}{c}\text { Speed of } \\
\text { sound }(\mathrm{m} / \mathrm{s})\end{array}$ & Z (MRayl) & $\begin{array}{c}\text { Attenuation } \\
(\mathrm{dB} / \mathrm{cm}) \text { at } 10 \mathrm{MHz}\end{array}$ & Reference \\
\hline BMF & $1.050-1.055$ & $1570-1595$ & $1.65-1.68$ & $1.5-2.2$ & 11 \\
\hline Liver & & $1568-1588$ & & $3.6-5.7$ & 31,32 \\
\hline TMM & & $1540 \pm 15$ & $1.60 \pm 0.16$ & 5 & 11 \\
\hline Ecoflex 00-10 & 1.06 & 1024 & 1.086 & 45.2 & \\
\hline Ecoflex 00-20 & 1.05 & 1043 & 1.074 & 33.9 & \\
\hline Ecoflex 00-30 & 1.05 & 1025 & 1.076 & 34.4 & \\
\hline Dragonskin & 1.07 & 1049 & 1.080 & 45.8 & \\
\hline Sortaclear18 & 1.07 & 1027 & 1.099 & 41.8 & \\
\hline Moldstar & 1.13 & 1051 & 1.082 & 57.6 & \\
\hline PDMS (Sylgard 184) & 1.05 & 1027 & 1.040 & 47.5 & \\
\hline
\end{tabular}

$\mathrm{TMM}=$ tissue-mimicking material; BMF = blood-mimicking fluid; PDMS = standard polydimethylsiloxane.

Monochromatic Superb Microvascular Imaging (mSMI) and Color Superb Microvascular Imaging (cSMI) are two microvascular Doppler modes developed specifically on the Aplio 500 scanner. ${ }^{30}$ They are based on an algorithm that allows visualization of low-velocity microvascular flow without injection of contrast agent. None of these three modes available on the Aplio 500 scanner, namely, PD, cSMI and mSMI, displayed the flow velocity.

Acquisition parameters were optimized and kept constant throughout the experiment: applied acoustic pressure was set to maximum (100\%), mechanical index (MI) to 1.1, gain to 88 , and dynamic range to $70 \mathrm{~dB}$. Parameters used for PD mode were: Pulse repetition frequency (Prf) 3.4 $\mathrm{kHz}$ (Velocity bar range 0.6), MI 1.5, 5 fps; parameters used for mSMI mode were: Prf $3.7 \mathrm{kHz}$ (Velocity bar range 0.4), MI 1.5, $37 \mathrm{fps,} \mathrm{SMI} \mathrm{7.2} \mathrm{MHz;} \mathrm{and} \mathrm{parameters} \mathrm{used} \mathrm{for} \mathrm{cSMI} \mathrm{mode}$ were: Prf 3.7 kHz (Velocity range bar 0.4), MI 1.5, 37 fps, SMI 7.2 MHz.

\section{Results}

The acoustic properties of the different silicone materials including Sylgard 184 PDMS (Dow Corning, Midland, Michigan) were measured and compared with the International Electrotechnical Commission flow test object standards ${ }^{11}$ (Table 1): none of the materials tested had acoustic properties matching the acoustic properties of tissue. The sound velocities were low ranging from 1024 to $1049 \mathrm{~m} / \mathrm{s}$, the acoustic impedance was about 1.1 MRayl and high attenuations between 34 and $57 \mathrm{~dB} / \mathrm{cm}$ were measured at $10 \mathrm{MHz}$. Among the tested materials, Ecoflex 00-30 and 00-20 had the lowest attenuation at $10 \mathrm{MHz}$, implying that for vessels placed at $2 \mathrm{~mm}$ depth, the attenuation was equivalent to $1.3 \mathrm{~cm}$ of tissue. Ecoflex 00-30 was ultimately chosen because it had a higher Shore $A$ hardness (a physical parameter measuring the hardness of a polymer material) according to the manufacturer and was thus easier to handle compared with Ecoflex 00-20.

For the flow studies, the setup makes it possible to use extremely slow flow rates around $50 \mu \mathrm{L} / \mathrm{min}$. To study the variability of the setup, the flow rate was measured for each channel and for each pressure over a period of three minutes. The measured flow rates as a function of applied pressure are shown in Figure 4 for the different channel diameters. For each channel and each pressure, the coefficient of variation was less than $1 \%$ over three minutes including 1800 measurement points. A linear relationship was observed between flow rate and pressure with a proportional coefficient ranging from 0.034 to $0.037 \mu \mathrm{L} \cdot \mathrm{min}^{-1} \cdot \mathrm{mbar}^{-1}\left(R^{2}>0.995\right)$, independent 
Grand-Perret et al.

of the channel size. This coefficient represented the hydrodynamic resistance provided by the 
Table 2. Comparison of Power Doppler and Superb Microvascular Imaging (Color and Monochrome) for Each Velocity and Each Channel Diameter.

\begin{tabular}{ccccccc}
\hline Channel & $\begin{array}{r}\text { Pressure } \\
(\mathrm{mbar})\end{array}$ & $\begin{array}{c}\text { Flow rate } \\
(\mu \mathrm{L} / \mathrm{min})\end{array}$ & $\begin{array}{c}\text { PD without } \\
\text { vibrations }\end{array}$ & $\begin{array}{c}\text { PD with } \\
\text { vibrations }\end{array}$ & $\begin{array}{c}\text { cSMl/mSMI } \\
\text { without vibrations }\end{array}$ & $\begin{array}{c}\text { cSMl/mSMI } \\
\text { with vibrations }\end{array}$ \\
\hline \multirow{6}{*}{$147 \mu \mathrm{m}$} & 1500 & 49 & NS & NS- & NS/++ & NS/++ \\
& 2000 & 71 & NS & NS- & NS/++ & NS/++ \\
& 3000 & 107 & NS & NS- & ++ & ++ \\
\multirow{4}{*}{$242 \mu \mathrm{m}$} & 4000 & 141 & NS & NS- & ++ & ++ \\
& 1500 & 55 & NS & - & ++ & ++ \\
& 2000 & 71 & NS & - & ++ & ++ \\
$318 \mu \mathrm{m}$ & 3000 & 105 & ++ & - & ++ & ++ \\
& 4000 & 137 & ++ & - & ++ & ++ \\
& 1500 & 57 & NS & - & ++ & ++ \\
$436 \mu \mathrm{m}$ & 2000 & 74 & ++ & - & ++ & ++ \\
& 3000 & 109 & ++ & - & ++ & ++ \\
& 4000 & 145 & ++ & - & ++ & ++ \\
& 1500 & 58 & ++ & - & ++ & ++ \\
& 2000 & 71 & ++ & - & ++ & ++ \\
\hline
\end{tabular}

Each video clip was rated "++" if signal and artifacts could be distinguished, "-" if not, "NS" if there was no signal and "NS-" if there were some artifacts outside the channel but no signal inside. PD = Power Doppler Mode; $\mathrm{cSMI}=$ Color Superb Microvascular Imaging; $\mathrm{mSMI}=$ Monochromatic Superb Microvascular Imaging .

whole circuit. The local resistance $R_{h}$ in a portion of channel of length $L$ and radius $R$ is given by the formula:

$$
R_{h}=\frac{8 \cdot \mu \cdot L}{\left(\pi \cdot R^{4}\right)},
$$

where $\mu$ is the viscosity of the BMF. The total resistance of the circuit is given by the sum of the local resistances. In our setup, the majority of the fluidic resistance was provided by the circuit upstream of the phantom, and the phantom channel only contributed to less than $5 \%$ of the total resistance. Therefore, the channel size in the phantom did not change significantly the total resistance. The corresponding velocities can be calculated from the flow rate and the diameter of the channel: a minimum velocity of $6 \mathrm{~mm} / \mathrm{s}$ is obtained with the $436 \mu \mathrm{m}$ tube at a flowrate of $58 \mu \mathrm{L} / \mathrm{min}$, and a maximum velocity of $142 \mathrm{~mm} / \mathrm{s}$ is obtained with the $147 \mu \mathrm{m}$ tube at 141 $\mu \mathrm{L} / \mathrm{min}$ flow rate.

The spectrum of the vibrations has been acquired. The amplitudes of the vibrations were less than $50 \mu \mathrm{m}$ at a low frequency of $24 \mathrm{~Hz}$.

Comparison of the results obtained with the PD and SMI (cSMI and mSMI) imaging modes was performed for each velocity and each channel diameter, as shown in Table 2.

For the smallest channel of $147 \mu \mathrm{m}$, and regardless of the presence of vibrations, the PD mode has no signal for flows between 49 and $141 \mu \mathrm{L} / \mathrm{min}$, while cSMI has no signal for flows between 49 and $71 \mu \mathrm{L} / \mathrm{min}$ and $\mathrm{mSMI}$ had signal for all tested flows.

In the absence of vibrations, for the larger channels ranging from 242 to $318 \mu \mathrm{m}$, the PD mode has no signal for slow flows of about 54 up to $75 \mu \mathrm{L} / \mathrm{min}$, unlike SMI modes. In the remaining 
Grand-Perretethetaf gest channel of $436 \mu \mathrm{m}$ and for higher flows in the smaller channels, it was possible to separate the signal from the artifacts in all imaging modes (Figure 5a, c, and e). 

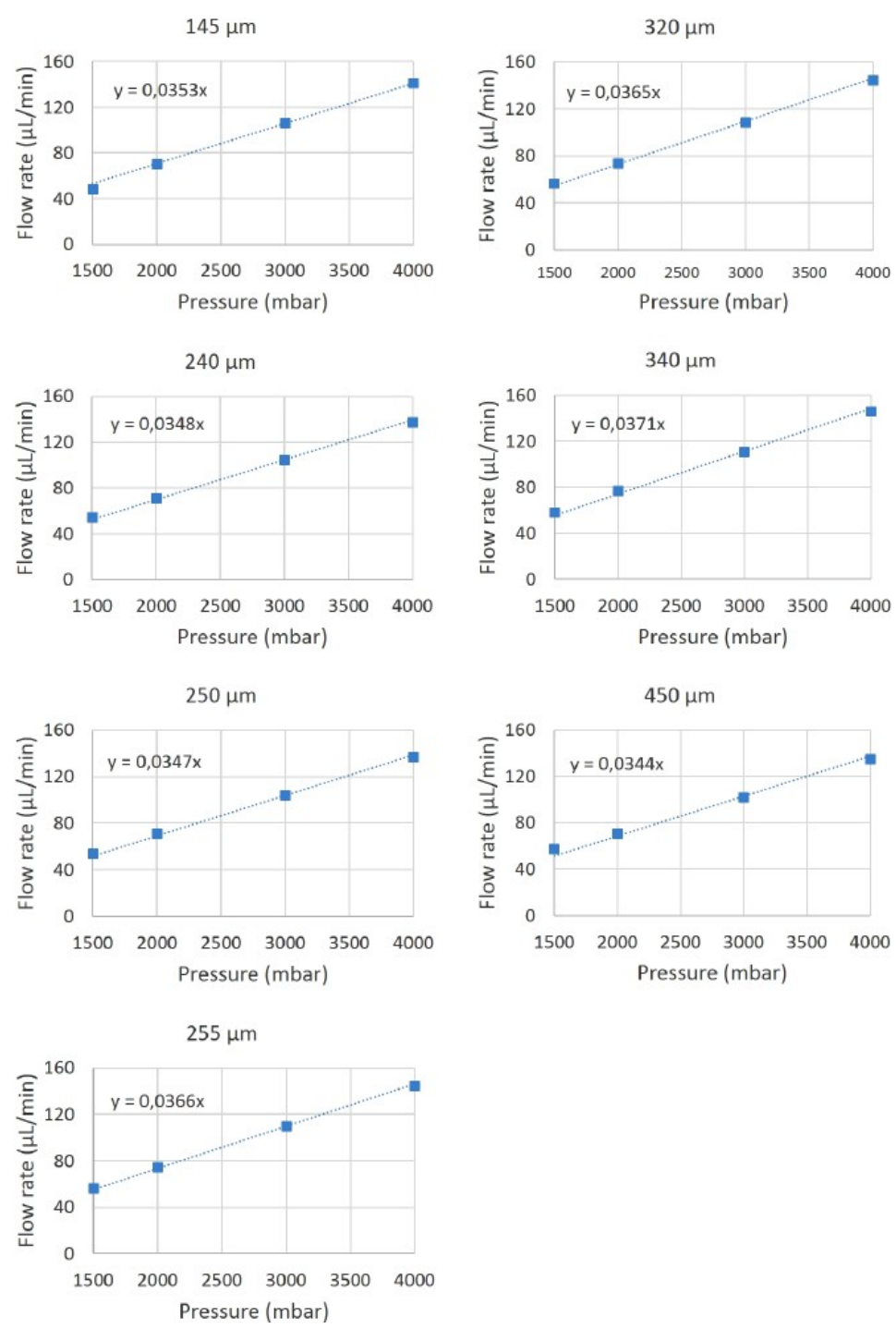

Figure 4. Flow rate as a function of applied pressure, for the seven channels. Standard deviation bars are not visible at this scale.

On the contrary, in the presence of vibrations, for larger channels ranging from 242 to $436 \mu \mathrm{m}$, the artifacts made it impossible to correctly visualize the signal in PD mode, but this was possible in the cSMI and mSMI modes (Figure $5 \mathrm{~b}$, d, and $\mathrm{f}$ ).

\section{Discussion}

We developed a novel flow phantom which can be used for microvascular assessment, a key parameter in tumor imaging. It was based on a silicone material (Ecoflex 00-30) including 


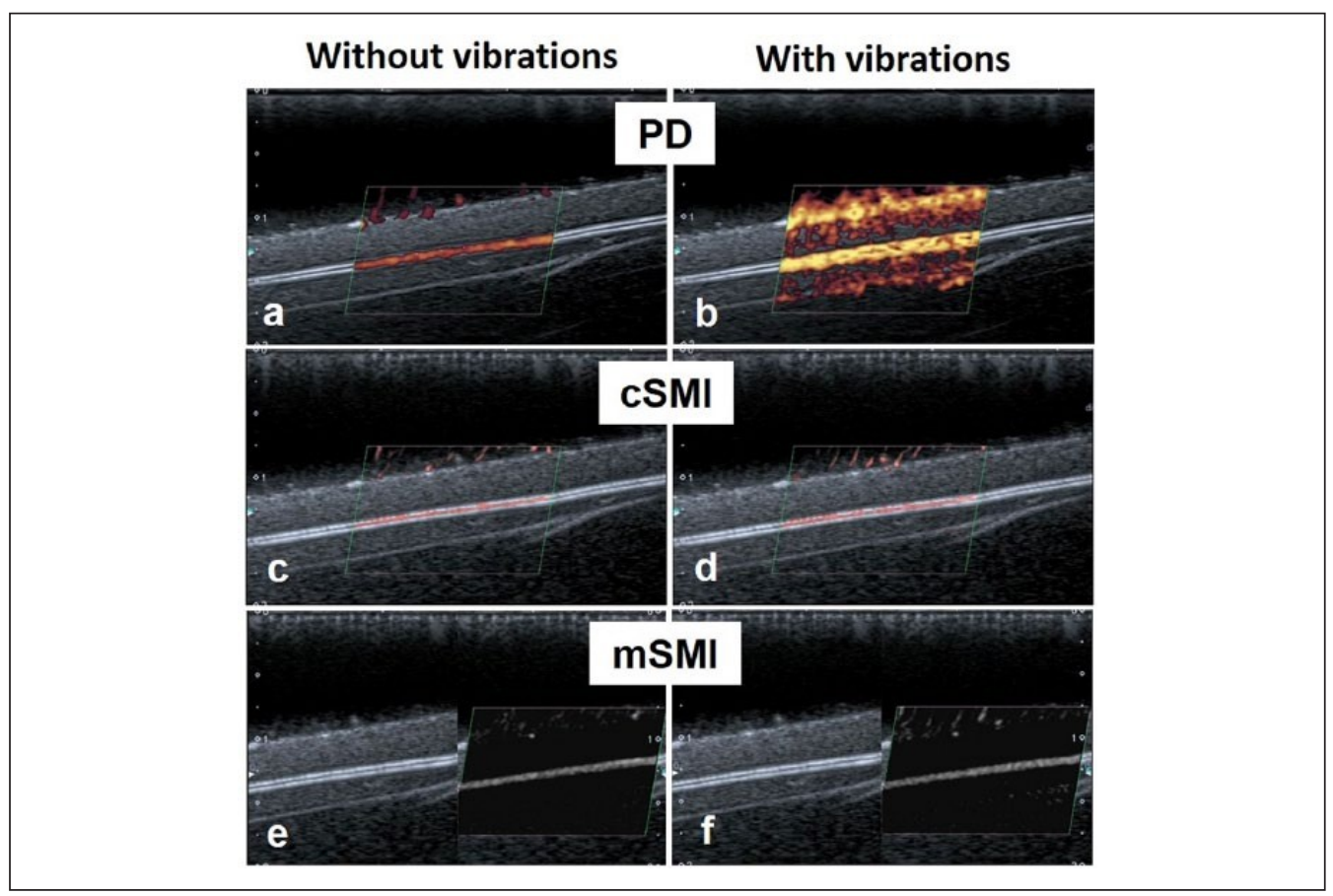

Figure 5. Ultrasound images of the $242 \mu \mathrm{m}$ channel at $750 \mathrm{mbar}$ of the flow phantom without (left) and with (right) vibrations. a, b: PD; c, d: cSMl; e, f: mSMI. Vascular signal is shown in the selected region of interest, displayed on top of black and white B-mode. In the case of monochrome Superb Microvascular Imaging, the vascular signal is shown in twin view display. PD = Power Doppler; cSMI = color Superb Microvascular Imaging; $\mathrm{mSMI}=$ monochrome Superb Microvascular Imaging.

circular channels of diameters ranging from 147 to $436 \mu \mathrm{m}$, which were easily made without photolithography in a clean room platform. The simple fabrication process made it easier to reproduce the procedure and thus make similar phantoms. A microfluidic pressure controlled setup enabled precise control and stable flow rates between 49 and $146 \mu \mathrm{L} / \mathrm{min}$. This device demonstrated that both the cSMI and mSMI modes could better filter mild vibrations than Power Doppler in channels from 147 to $436 \mu \mathrm{m}$ with velocities ranging from $6 \mathrm{~mm} / \mathrm{s}$ to $142 \mathrm{~mm} / \mathrm{s}$.

The silicone used in this phantom, Ecoflex 00-30, was the best compromise among the silicone materials that were acoustically characterized. It shared the advantages and the drawbacks of silicones. Indeed, it is easily molded and has a long shelf life compared with agarose phantoms. However, the speed of sound at ultrasound frequencies at $10 \mathrm{MHz}$ was significantly lower than in biological tissues, resulting in a distortion in the displayed image, because the speed of sound cannot be adjusted on the clinical ultrasound imaging device. However, this was not an important limit since the region of interest was not the surrounding material but the channel itself. This channel was filled with BMF having the acoustical properties required by the International Electrotechnical Committee, ${ }^{11}$ so the image of the channel was displayed with cor- rect dimensions. Moreover, the attenuation of Ecoflex, another crucial acoustical property, was not a limiting factor to detect ultrasound signal in the channel placed $2 \mathrm{~mm}$ under the surface ofthe phantom. Indeed, $2 \mathrm{~mm}$ of Ecoflex is equivalent in attenuation to $1.4 \mathrm{~cm}$ of TMM. This is consistent with the depth of the regions of interest used in clinical examinations made with this high-frequency probe. As regard to mechanical properties, Ecoflex enables to make microflow 
geometries whereas widely used agarose phantoms cannot. PDMS is another silicone material known to be used to make microfluidic chip featuring microflow channels, and has thus been included in our study. PDMS had a higher acoustic attenuation than Ecoflex, which would imply using under $1 \mathrm{~mm}$ material above the channel to measure the same ultrasound signal as over 1.4 $\mathrm{cm}$ of TMM. This thickness is a limit to mechanical strength of the channel. Furthermore, it is more expensive and has a more complex fabrication process including a dedicated mixing device to avoid gas bubbles and postcuring equipment. Being the standard material used in microfluidics, ${ }^{33,34}$ PDMS has been used to make various types of chips for ultrasound imaging characterizations. Kim et al. proposed chips suitable to mimic teeth ${ }^{21}$ including a glass slide that mimicked teeth enamel. The channel dimensions and flows were similar to those used in our study. The design we developed had no glass slide, and thus enabled better penetration of the ultrasound waves. Desailly et al. have used microfluidic chips to study ultrafast ultrasound localization microscopy. ${ }^{18,20}$ The channel dimension of $80 \times 60 \mu \mathrm{m}^{2}$ was smaller than our channels, but the velocities were significantly faster $(500 \mu \mathrm{L} / \mathrm{min}$ flow rate). Moreover, the flow within the chip was maintained using a syringe pump. The stepper motor inside the syringe pump is reported to be a source of fluctuations in the flow rate ${ }^{35}$ and the use of a syringe pump is limited to a small reservoir to generate precise slow flow rates. By contrast, our use of a microfluidic flow pressure controller with less than $1 \%$ coefficient of variation of relative flow rate allowed us to have a stable flow using a large volume of BMF.

The interest of this setup was illustrated by performing ultrasound imaging on micro channels embedded in Ecoflex. First, the ultrasound image in B-mode was able to display the channels for every dimension. It could be noticed on the ultrasound images that artifacts were present at the interfaces between the silicone surrounding material and the channels filled with BMF. They were probably due to strong reflection because of acoustic impedance mismatches between the silicone rubber and the BMF as previously reported in a wall-less design similar to ours. ${ }^{20}$ Furthermore, it has been reported that channel size was overestimated by ultrasound, and that this measure had high variability ${ }^{16}$ : the diameter of a $200 \mu \mathrm{m}$ channel was measured to be $600 \mu \mathrm{m}$ with a standard deviation of $400 \mu \mathrm{m}$, which makes an overestimation of $200 \%$. That is why our velocities were calculated from the channel sizes measured precisely on the macroscope. Second, ultrasound modes dedicated to microflow imaging, precisely PD and SMI, were investigated, especially their performances with the presence of vibrations. We addressed this issue since the physiological movements frequently encountered during clinical examinations, mainly breathing of the patient and heartbeats, are known to cause image artifacts. The applied vibrations had a higher frequency than those of physiological movements (around a few Hertz), ${ }^{36,37}$ but were expected to have less impact on the image since their amplitude was much smaller than physiological motion: less than $50 \mu \mathrm{m}$ compared with a few millimeters. ${ }^{38}$ Our experimental setup showed that Power Doppler was not able to detect the signal in the small channel of $147 \mu \mathrm{m}-$ diameter, suggesting a limitation in spatial resolution, and in bigger channels for the smallestflow rates, suggesting a limitation in sensitivity. In the presence of vibrations, artifacts covered up the signal: for the $147 \mu \mathrm{m}$ channel, there was no signal in the channel but there were some artifacts in the surrounding material; for larger diameters, the artifacts could not be distinguishedfrom the signal.

Overall, our new microvascular flow phantom setup demonstrated that the SMI modes exhibited good sensitivity in the detection of microvessel flow distribution patterns, as observed and described in some clinical cases. ${ }^{8,9}$ Moreover, the PD mode was very sensitive to the vibrations we applied, limiting the detection of microcirculation. This microvascular flow setup could also be used for dynamic contrast-enhanced ultrasound modes to develop new imaging modalities, ${ }^{39,40}$ new transducers ${ }^{41}$ or new quantification methods. ${ }^{42}$ 


\section{Conclusion}

We designed and fabricated a new prototype of flow phantom based on a microfluidic pressure controlled setup, with circular channels of dimensions ranging from $147 \mu \mathrm{m}$ to $436 \mu \mathrm{m}$ surrounded by an easy-to-handle, acoustic compatible soft silicone material (Ecoflex 00-30). It enabled precise control of 49 to $146 \mu \mathrm{L} / \mathrm{min}$ flow rates with less than $1 \%$ coefficient of variation during the acquisition. Experiments led using microflow Doppler imaging modes have been able to demonstrate better visualization in SMI compared with PD, in accordance with what was clinically reported, due to reduced motion artifacts in the SMI mode versus the PD. Future developments include the use of a less ultrasound-absorbing material and more com- plex channel geometries.

\section{Acknowledgments}

The authors thank the optical Platform PFIC who provided help for channel diameter measurements.

\section{Declaration of Conflicting Interests}

The author(s) declared no potential conflicts of interest with respect to the research, authorship, and/or publication of this article.

\section{Funding}

The author(s) disclosed receipt of the following financial support for the research, authorship, and/or publication of this article: This study was supported by grants from the research program in physics, mathematics and engineering sciences applied to cancer research, of the French Plan Cancer 2013, the Canceropôle Ilede-France, and the ARC foundation.

\section{References}

1. Lanza GA, Camici PG, Galiuto L, Niccoli G, Pizzi C, Di Monaco A, et al. Methods to investigate coronary microvascular function in clinical practice: J Cardiovasc Med. 2013;14(1):1-18.

2. Pries AR, Habazettl H, Ambrosio G, Hansen PR, Kaski JC, Schachinger V, et al. A review of methods for assessment of coronary microvascular disease in both clinical and experimental settings. Cardiovasc Res. 2008;80(2):165-74.

3. Lassau N, Bonastre J, Kind M, Vilgrain V, Lacroix J, Cuinet M, et al. Validation of dynamic contrastenhanced ultrasound in predicting outcomes of antiangiogenic therapy for solid tumors: the French multicenter support for innovative and expensive techniques study. Invest Radiol. 2014;49(12):794800 .

4. Carmeliet P, Jain RK. Molecular mechanisms and clinical applications of angiogenesis. Nature. 2011;473(7347):298-307.

5. O'Connor JPB, Aboagye EO, Adams JE, Aerts HJWL, Barrington SF, Beer AJ, et al. Imaging biomarker roadmap for cancer studies. Nat Rev Clin Oncol. 2017;14(3):169-86.

6. Lassau N, Koscielny S, Opolon P, De Baere T, Peronneau P, Leclere Jé, et al. Evaluation of con- trastenhanced color Doppler ultrasound for the quantification of angiogenesis in vivo. Invest Radiol. 2001;36(1):50-55.

7. Desailly Y, Tissier A-M, Correas J-M, Wintzenrieth F, Tanter M, Couture O. Contrast enhanced ultrasound by real-time spatiotemporal filtering of ultrafast images. Phys Med Biol. 2017;62(1):31-42.

8. Forsberg F, Machado P, Segal S, Okamura Y, Guenette G, Rapp C, et al. Microvascular blood flow in the thyroid: preliminary results with a novel imaging technique. IEEE Int Ultrason Symp Proc. 2014;2237-40.

9. Gabriel M, Tomczak J, Snoch-Ziółkiewicz M, Dzieciuchowicz Ł, Strauss E, Oszkinis G. Comparison of superb micro-vascular ultrasound imaging (SMI) and contrast-enhanced ultrasound (CEUS) for 
detection of endoleaks after endovascular aneurysm repair (EVAR). Am J Case Rep. 2016;17:43-46.

10. Yongfeng Z, Ping Z, Wengang L, Yang S, Shuangming T. Application of a novel microvascular imaging technique in breast lesion evaluation. Ultrasound Med Biol. 2016;42(9):2097-105.

11. International Electrotechnical Commission. Ultrasonics-Flow measurement systems-Flow test object, IEC 61685. Geneva; 2001. Available from https://webstore.iec.ch/publication/5721

12. Franchi-Abella S, Elie C, Correas J-M. Ultrasound elastography: advantages, limitations and artefacts of the different techniques from a study on a phantom. Diagn Interv Imaging. 2013;94(5):497-501.

13. Ho CK, Chee AJY, Yiu BYS, Tsang ACO, Chow KW, Yu ACH. Wall-less flow phantoms with tortuous vascular geometries: design principles and a patient-specific model fabrication example. IEEE Trans Ultrason Ferroelectr Freq Control. 2017;64(1):25-38.

14. Yiu BYS, Yu ACH. Spiral flow phantom for ultrasound flow imaging experimentation. IEEE Trans Ultrason Ferroelectr Freq Control. 2017;64(12):1840-8.

15. Browne JE. A review of Doppler ultrasound quality assurance protocols and test devices. Phys Med. 2014;30(7):742-51.

16. Kenwright DA, Laverick N, Anderson T, Moran CM, Hoskins PR. Wall-less flow phantom for highfrequency ultrasound applications. Ultrasound Med Biol. 2015;41(3):890-7.

17. Less JR, Skalak TC, Sevick EM, Jain RK. Microvascular architecture in a mammary carcinoma: branching patterns and vessel dimensions. Cancer Res. 1991;51(1):265-73.

18. Camfferman FA, Ecury-Goossen GM, La Roche JE, de Jong N, van't Leven W, Vos HJ, et al. Calibrating Doppler imaging of preterm intracerebral circulation using a microvessel flow phantom. Front Hum Neurosci. 2015;8(1068):1-8.

19. Hindle AJ, Perkins AC. A perfusion phantom for the evaluation of ultrasound contrast agents. Ultrasound Med Biol. 1994;20(3):309-14.

20. Desailly Y, Pierre J, Couture O, Tanter M. Resolution limits of ultrafast ultrasound localization microscopy. Phys Med Biol. 2015;60(22):8723-40.

21. Kim D, Park S-H. A microfluidics-based pulpal arteriole blood flow phantom for validation of doppler ultrasound devices in pulpal blood flow velocity measurement. J Endod. 2016;42(11):1660-6.

22. Oates CP. Towards an ideal blood analogue for Doppler ultrasound phantoms. Phys Med Biol. 1991;36(11):1433.

23. Ramnarine KV, Nassiri DK, Hoskins PR, Lubbers J. Validation of a new blood-mimicking fluid for use in Doppler flow test objects. Ultrasound Med Biol. 1998;24(3):451-9.

24. Samavat H, Evans JA. An ideal blood mimicking fluid for Doppler ultrasound phantoms. J Med Phys Assoc Med Phys India. 2006;31(4):275-8.

25. AIUM. Methods for specifying acoustic properties of tissue mimicking phantoms and objects, Stage I, Laurel, MD: American Institute of Ultrasound in Medicine Technical Standard Committee, 1995. Available from https://trove.nla.gov.au/version/42746800

26. Sun C, Pye SD, Browne JE, Janeczko A, Ellis B, Butler MB, et al. The speed of sound and attenuation of an IEC agar-based tissue-mimicking material for high frequency ultrasound applications. Ultrasound Med Biol. 2012;38(7):1262-70.

27. Kenwright DA, Sadhoo N, Rajagopal S, Anderson T, Moran CM, Hadoke PW, et al. Acoustic assessment of a konjac-carrageenan tissue-mimicking material at 5-60 MHz. Ultrasound Med Biol. 2014;40(12):2895-2902.

28. Marczak W. Water as a standard in the measurements of speed of sound in liquids. J Acoust Soc Am. 1997;102(5):2776-9.

29. He P, Zheng J. Acoustic dispersion and attenuation measurement using both transmitted and reflected pulses. Ultrasonics. 2001;39(1):27-32.

30. Hata J. Seeing the unseen new techniques in vascular imaging. 2014. Available from https://us.medical. canon/download/aplio-500-wp-smi-seeing-the-unseen

31. Chen CF, Robinson DE, Wilson LS, Griffiths KA, Manoharan A, Doust BD. Clinical sound speed measurement in liver and spleen in vivo. Ultrason Imaging. 1987;9(4):221-35.

32. Garra BS, Insana MF, Shawker TH, Russell MA. Quantitative estimation of liver attenuation and echogenicity: normal state versus diffuse liver disease. Radiology. 1987 Jan;162(1):61-7. 
33. Anderson JR, Chiu DT, Wu H, Schueller OJ, Whitesides GM. Fabrication of microfluidic systems in poly (dimethylsiloxane). Electrophoresis. 2000;21(1):27-40.

34. Whitesides GM. The origins and the future of microfluidics. Nature. 2006;442(7101):368.

35. Li Z, Mak SY, Sauret A, Shum HC. Syringe-pump-induced fluctuation in all-aqueous microfluidic system implications for flow rate accuracy. Lab Chip. 2014;14(4):744-9.

36. Thakral A, Wallace J, Tomlin D, Seth N, Thakor NV. Surgical Motion Adaptive Robotic Technology (S.M.A.R.T): taking the motion out of physiological motion. In: Niessen WJ, Viergever MA, eds. Medical Image Computing and Computer-Assisted Intervention - MICCAI 2001: 4th International Conference Utrecht, The Netherlands, October 14-17, 2001 Proceedings [Internet]. Berlin, Heidelberg: Springer; 2001;317-25. Available from https://doi.org/10.1007/3-540-45468-3_38.

37. Ginhoux R, Gangloff J, de Mathelin M, Soler L, Sanchez MMA, Marescaux J. Active filtering of physiological motion in robotized surgery using predictive control. IEEE Trans Robot. 2005;21(1):67-79.

38. Kolen AF, Miller NR, Ahmed EE, Bamber JC. Characterization of cardiovascular liver motion for the eventual application of elasticity imaging to the liver in vivo. Phys Med Biol. 2004;49(18):4187-206.

39. Eisenbrey JR, Sridharan A, Liu J-B, Forsberg F. Recent experiences and advances in contrast-enhanced subharmonic ultrasound. Biomed Res Int. 2015;Article ID 640397.

40. Errico C, Osmanski B-F, Pezet S, Couture O, Lenkei Z, Tanter M. Transcranial functional ultrasound imaging of the brain using microbubble-enhanced ultrasensitive Doppler. NeuroImage. 2016;124:752-61.

41. Fouan D, Bouakaz A. Investigation of classical pulse sequences for contrast-enhanced ultrasound imaging with a cMUT probe. IEEE Trans Ultrason Ferroelectr Freq Control. 2016;63(10):1496-504.

42. Cheung WK, Gujral DM, Shah BN, Chahal NS, Bhattacharyya S, Cosgrove DO, et al. Attenuation correction and normalisation for quantification of contrast enhancement in ultrasound images of carotid arteries. Ultrasound Med Biol. 2015;41(7):1876-83. 